\title{
CM EVALUATIONS OF THE GOSWAMI-SUN SERIES
}

\author{
MADELINE LOCUS DAWSEY* AND KEN ONO
}

\begin{abstract}
In recent work, Sun constructed two $q$-series, and he showed that their limits as $q \rightarrow 1$ give new derivations of the Riemann-zeta values $\zeta(2)=\pi^{2} / 6$ and $\zeta(4)=\pi^{4} / 90$. Goswami extended these series to an infinite family of $q$-series, which he analogously used to obtain new derivations of the evaluations of $\zeta(2 k) \in \mathbb{Q} \cdot \pi^{2 k}$ for every positive integer $k$. Since it is well known that $\Gamma\left(\frac{1}{2}\right)=\sqrt{\pi}$, it is natural to seek further specializations of these series which involve special values of the $\Gamma$-function. Thanks to the theory of complex multiplication, we show that the values of these series at all CM points $\tau$, where $q:=e^{2 \pi i \tau}$, are algebraic multiples of specific ratios of $\Gamma$-values. In particular, classical formulas of Ramanujan allow us to explicitly evaluate these series as algebraic multiples of powers of $\Gamma\left(\frac{1}{4}\right)^{4} / \pi^{3}$ when $q=e^{-\pi}, e^{-2 \pi}$.
\end{abstract}

\section{Introduction and Statement of Results}

Recently, Sun [10] obtained two $q$-series identities which allowed him to prove that

$$
\lim _{\substack{q \rightarrow 1 \\|q|<1}}(1-q)^{2} \sum_{n=0}^{\infty} \frac{q^{n}\left(1+q^{2 n+1}\right)}{\left(1-q^{2 n+1}\right)^{2}}=\frac{3}{2} \zeta(2)=\frac{\pi^{2}}{4}
$$

and

$$
\lim _{\substack{q \rightarrow 1 \\|q|<1}}(1-q)^{4} \sum_{n=0}^{\infty} \frac{q^{2 n}\left(1+4 q^{2 n+1}+q^{4 n+2}\right)}{\left(1-q^{2 n+1}\right)^{4}}=\frac{45}{8} \zeta(4)=\frac{\pi^{4}}{16} .
$$

Sun's formulas lead to the natural question: Are these $q$-series a glimpse of an infinite family that offers new derivations for the evaluations of $\zeta(2 k)$ for all positive integers $k$ ? Goswami elegantly answered this problem in [5]; he defined a natural family of identities whose limits as $q \rightarrow 1$ with $|q|<1$ give Euler's formula for the Riemann-zeta values at all even integers.

These results have been described as $q$-analogues of Euler's identities for $\zeta(2 k)$. Here we offer further support of this view. Namely, to be a strong $q$-analogue, one hopes for further specializations of $q$ which are expressions in related special functions. We address this question by observing that $\zeta(2 k) \in \mathbb{Q} \cdot \pi^{2 k}=\mathbb{Q} \cdot \Gamma\left(\frac{1}{2}\right)^{4 k}$, and we ask if Goswami's series have evaluations involving algebraic multiples of naturally corresponding $\Gamma$-values. We show that this is indeed the case, thanks to the theory of complex multiplication and modular forms.

In order to state our results, we first recall the $q$-series that Goswami assembled which extended Sun's original identities into an infinite family. Throughout, $k$ is a positive integer. If we denote the Stirling numbers of the second kind by $\left\{\begin{array}{l}n \\ k\end{array}\right\}$, then we define $a_{k}(m)$ and $b_{k}(\ell)$

2010 Mathematics Subject Classification. 11B99, 11Mxx, 11F11.

Key words and phrases. q-analogue, Riemann-zeta values, Gamma-values.

*This author was previously known as Madeline Locus. 
by

$$
\begin{aligned}
a_{k}(m) & :=\sum_{j=0}^{2 k-1} j !(-1)^{j}\left\{\begin{array}{c}
2 k-1 \\
j
\end{array}\right\}\left(\begin{array}{c}
j \\
m
\end{array}\right), \\
b_{k}(\ell) & :=\sum_{m=0}^{2 k-1}(-1)^{m} a_{k}(m)\left(\begin{array}{c}
2 k-m-1 \\
\ell
\end{array}\right) \in \mathbb{Z} .
\end{aligned}
$$

Using these quantities, we define the degree $2 k-2$ polynomial

$$
P_{2 k-2}^{e}(z):=\sum_{\ell=1}^{2 k-1}(-1)^{\ell} b_{k}(\ell) z^{\ell-1},
$$

and the degree $4 k-2$ polynomial

$$
P_{4 k-2}^{o}(z):=(1+z)^{2 k} P_{2 k-2}^{e}(z)-2^{2 k-1} z P_{2 k-2}^{e}\left(z^{2}\right) .
$$

For notational convenience, we define Goswami's $q$-series as follows.

$$
\mathcal{G}_{2 k}(q):= \begin{cases}\sum_{n=0}^{\infty} \frac{q^{2 n+1} P_{4 k-2}^{o}\left(q^{2 n+1}\right)}{\left(1-q^{4 n+2}\right)^{2 k}}, & \text { if } k \text { is odd } . \\ 2^{2 k-1} \sum_{n=0}^{\infty} \frac{q^{4 n+2} P_{2 k-2}^{e}\left(q^{4 n+2}\right)}{\left(1-q^{4 n+2}\right)^{2 k}}, & \text { if } k \text { is even, }\end{cases}
$$

Remark. When $k=1$ and $k=2$, these are essentially Sun's $q$-series. A critical feature of the results obtained here is that the $\mathcal{G}_{2 k}(q)$ are holomorphic modular forms on $\Gamma_{0}(4)$ of integer weight $2 k$.

As usual, we let $\overline{\mathbb{Q}}$ denote the algebraic closure of the field of rational numbers. Suppose that $D<0$ is the fundamental discriminant of the imaginary quadratic field $\mathbb{Q}(\sqrt{D})$. Let $h(D)$ denote the class number of $\mathbb{Q}(\sqrt{D})$, and define $h^{\prime}(D):=1 / 3($ resp. $1 / 2)$ when $D=-3$ (resp. -4 ), and $h^{\prime}(D):=h(D)$ when $D<-4$. We then let

$$
\omega_{D}:=\frac{1}{\sqrt{\pi}}\left(\prod_{j=1}^{|D|-1} \Gamma\left(\frac{j}{|D|}\right)^{\chi_{D}(j)}\right)^{\frac{1}{2 h^{\prime}(D)}}
$$

where $\chi_{D}(\bullet):=\left(\frac{D}{\bullet}\right)$. In terms of this notation, we obtain the following theorem.

Theorem 1.1. If $D<0$ is a fundamental discriminant and $\tau \in \mathbb{H} \cap \mathbb{Q}(\sqrt{D})$, then

$$
\mathcal{G}_{2 k}\left(e^{2 \pi i \tau}\right) \in \overline{\mathbb{Q}} \cdot \omega_{D}^{2 k}
$$

Thanks to classical formulas of Ramanujan [1], it is simple to explicitly evaluate $\mathcal{G}_{2 k}\left(e^{-\pi}\right)$ and $\mathcal{G}_{2 k}\left(e^{-2 \pi}\right)$. To make this precise, we define the rational number 1

$$
\mathcal{Z}(2 k):=-\frac{(-16)^{k} B_{2 k}\left(4^{k}-1\right)}{8 k}=4^{k-1}\left(4^{k}-1\right)(2 k) ! \cdot \frac{\zeta(2 k)}{\pi^{2 k}},
$$

\footnotetext{
${ }^{1}$ In [5], Goswami refers to $\mathcal{Z}(2 k)$ as $d_{k}$. We use $\mathcal{Z}(2 k)$ to emphasize that these numbers are simple rational multiples of $\zeta(2 k) / \pi^{2 k}$.
} 
where $B_{2 k}$ is the index $2 k$ Bernoulli number. Furthermore, we let $(a ; q)_{\infty}:=\prod_{n \geq 0}\left(1-a q^{n}\right)$ denote the usual infinite $q$-Pochhammer symbol. If $k \geq 2$, then define $\alpha_{2 k}(1), \ldots, \alpha_{2 k}(k-1)$ to be the unique rational numbers satisfying

$$
\sum_{j=1}^{k-1} \alpha_{2 k}(j) \cdot \frac{q^{j}\left(q^{4} ; q^{4}\right)_{\infty}^{16 j}(q ; q)_{\infty}^{8 j}}{\left(q^{2} ; q^{2}\right)_{\infty}^{24 j}}=\left(\mathcal{G}_{2 k}(q)-\mathcal{Z}(2 k) \cdot \frac{q^{k}\left(q^{4} ; q^{4}\right)_{\infty}^{8 k}}{\left(q^{2} ; q^{2}\right)_{\infty}^{4 k}}\right) \cdot \frac{(q ; q)_{\infty}^{8 k}\left(q^{4} ; q^{4}\right)_{\infty}^{8 k}}{\left(q^{2} ; q^{2}\right)_{\infty}^{20 k}}
$$

Since the $j$ th summand on the left is of the form $\alpha_{2 k}(j) q^{j}+O\left(q^{j+1}\right)$, the $\alpha_{2 k}(j)$ are easily computed by diagonalization. In the case where $k=1$, there simply are no $\alpha_{2 k}(j)$ numbers. In terms of this notation, we obtain the following corollary.

Corollary 1.2. If $k$ is a positive integer and $a:=\sqrt{2}-1$, then

$$
\begin{aligned}
\mathcal{G}_{2 k}\left(e^{-\pi}\right) & =\left(\frac{\mathcal{Z}(2 k)}{2^{7 k}}+\frac{1}{2^{2 k}} \sum_{j=1}^{k-1} \frac{\alpha_{2 k}(j)}{2^{5 j}}\right) \cdot\left(\frac{\Gamma\left(\frac{1}{4}\right)^{4}}{\pi^{3}}\right)^{k}, \\
\mathcal{G}_{2 k}\left(e^{-2 \pi}\right) & =\left(\frac{\mathcal{Z}(2 k) a^{2 k}}{2^{9 k}}+\frac{1}{2^{5 k} a^{2 k}} \sum_{j=1}^{k-1} \frac{\alpha_{2 k}(j) a^{4 j}}{2^{4 j}}\right) \cdot\left(\frac{\Gamma\left(\frac{1}{4}\right)^{4}}{\pi^{3}}\right)^{k} .
\end{aligned}
$$

Examples. Here we illustrate Corollary 1.2 for $k=3$ and 4 . If $k=3$, then we have that

$$
\begin{aligned}
\mathcal{G}_{6}\left(e^{-\pi}\right) & =\left(\frac{\mathcal{Z}(6)}{2^{21}}+\frac{1}{2^{12}}\right) \cdot\left(\frac{\Gamma\left(\frac{1}{4}\right)^{4}}{\pi^{3}}\right)^{3} \\
\mathcal{G}_{6}\left(e^{-2 \pi}\right) & =\left(\frac{\mathcal{Z}(6)(\sqrt{2}-1)^{6}}{2^{27}}+\frac{1-(\sqrt{2}-1)^{4}}{2^{19}(\sqrt{2}-1)^{2}}\right) \cdot\left(\frac{\Gamma\left(\frac{1}{4}\right)^{4}}{\pi^{3}}\right)^{3} .
\end{aligned}
$$

If $k=4$, then we have that

$$
\begin{aligned}
\mathcal{G}_{8}\left(e^{-\pi}\right) & =\left(\frac{\mathcal{Z}(8)}{2^{28}}+\frac{1}{2^{12}}\right) \cdot\left(\frac{\Gamma\left(\frac{1}{4}\right)^{4}}{\pi^{3}}\right)^{4}, \\
\mathcal{G}_{8}\left(e^{-2 \pi}\right) & =\left(\frac{\mathcal{Z}(8)(\sqrt{2}-1)^{8}}{2^{36}}+\frac{1-(\sqrt{2}-1)^{4}}{2^{21}}\right) \cdot\left(\frac{\Gamma\left(\frac{1}{4}\right)^{4}}{\pi^{3}}\right)^{4} .
\end{aligned}
$$

These examples will be explained further in Section 5 ,

This paper is organized as follows. In Section 2, we recall the Goswami-Sun identities and the relation between $\mathcal{G}_{2 k}(q)$ and modular forms. In Section 3, we recall essential facts about modular forms, and in Section 4, we use these results to prove Theorem 1.1 and Corollary 1.2. In Section 5, we conclude with a discussion of the examples given above.

\section{ACKNOWLEDGEMENTS}

We thank Krishnaswami Alladi and Ankush Goswami for their beautiful ideas and contributions. We also thank Zhi-Wei Sun for inspiring this work. 


\section{The Goswami-Sun Identities}

We now recall Goswami's work. Let $T_{n}=n(n+1) / 2$ denote the $n$th triangular number, and define the generating function of $T_{n}$ to be

$$
\psi(q):=\sum_{n \geq 0} q^{T_{n}}
$$

Then Goswami [5, Theorems 3.1 and 3.2] proves the following theorem.

Theorem 2.1. For any positive integer $k$, we have that

$$
T_{2 k}(\tau):=\mathcal{G}_{2 k}(q)-\mathcal{Z}(2 k) \cdot q^{k} \psi\left(q^{2}\right)^{4 k}
$$

is the Fourier expansion of a weight $2 k$ cusp form on $\Gamma_{0}(4)$, where $q:=e^{2 \pi i \tau}$ and $\tau \in \mathbb{H}$.

In Section 3, we apply the theory of modular forms and complex multiplication to study $T_{2 k}(\tau)$ and $q^{k} \psi\left(q^{2}\right)^{4 k}$ at all CM points.

\section{Some Facts about Modular Forms}

Here we recall some basic facts about modular forms.

3.1. CM Values of Modular Forms. In Goswami's work [5], the limit of the $q$-series identity in Theorem 2.1 as $q \rightarrow 1$ gives the constant term of a weight $2 k$ Eisenstein series, which is described in terms of $\zeta$-values. Our work depends on the values of modular forms at CM points.

Classically, the Chowla-Selberg formula [3] was developed in order to evaluate the Dedekind eta-function

$$
\eta(\tau):=q^{1 / 24} \prod_{n=1}^{\infty}\left(1-q^{n}\right)
$$

at CM points whose discriminants are fundamental discriminants. This was refined by van der Poorten and Williams [9, Theorem 9.3], who gave a closed formula for values of $\eta(\tau)$ in which $\tau$ is still required to be a CM point whose discriminant is fundamental. More generally, we have the following theorem (for example, see p. 84 of [2]) regarding evaluations of all modular forms at all CM points.

Theorem 3.1. Suppose that $D<0$ is the fundamental discriminant of the imaginary quadratic field $\mathbb{Q}(\sqrt{D})$. Then the number $\Omega_{D} \in \mathbb{C}^{*}$ defined by

$$
\Omega_{D}:=\frac{1}{\sqrt{2 \pi|D|}}\left(\prod_{j=1}^{|D|-1} \Gamma\left(\frac{j}{|D|}\right)^{\chi_{D}(j)}\right)^{\frac{1}{2 h^{\prime}(D)}}
$$

has the property that $f(\tau) \in \overline{\mathbb{Q}} \cdot \Omega_{D}^{k}$ for all $\tau \in \mathbb{H} \cap \mathbb{Q}(\sqrt{D})$, all $k \in \mathbb{Z}$, and all modular forms $f$ of weight $k$ with algebraic Fourier coefficients.

In the special cases of the CM points $\tau \in\{i / 2, i, 2 i, 4 i\}$, Theorem 3.1 can be made explicit using the following formulas of Ramanujan (see p. 326 of [1]),

$$
\begin{aligned}
& f\left(-e^{-\pi}\right)=\frac{\pi^{\frac{1}{4}} e^{\frac{\pi}{24}}}{2^{\frac{3}{8}} \Gamma\left(\frac{3}{4}\right)}, \\
& f\left(-e^{-2 \pi}\right)=\frac{\pi^{\frac{1}{4}} e^{\frac{\pi}{12}}}{2^{\frac{1}{2}} \Gamma\left(\frac{3}{4}\right)},
\end{aligned}
$$




$$
\begin{aligned}
& f\left(-e^{-4 \pi}\right)=\frac{\pi^{\frac{1}{4}} e^{\frac{\pi}{6}}}{2^{\frac{7}{8}} \Gamma\left(\frac{3}{4}\right)}, \\
& f\left(-e^{-8 \pi}\right)=\frac{\pi^{\frac{1}{4}}(\sqrt{2}-1)^{\frac{1}{4}} e^{\frac{\pi}{3}}}{2^{\frac{21}{16}} \Gamma\left(\frac{3}{4}\right)},
\end{aligned}
$$

where $f(-q):=\prod_{n>1}\left(1-q^{n}\right)$. The above formulas can be rewritten in terms of the Dedekind eta-function by noticing that $\eta(\tau)=q^{1 / 24} f(-q)$. By applying the functional equation of the $\Gamma$-function, namely $\Gamma(1-z) \Gamma(z)=\pi / \sin (\pi z)$ for $z \notin \mathbb{Z}$, in terms of

$$
\Omega_{-4}=\frac{1}{2 \sqrt{2 \pi}} \cdot \frac{\Gamma\left(\frac{1}{4}\right)}{\Gamma\left(\frac{3}{4}\right)}
$$

we obtain

$$
\eta(i / 2)=2^{\frac{1}{8}} \cdot \Omega_{-4}^{\frac{1}{2}}, \quad \eta(i)=\Omega_{-4}^{\frac{1}{2}}, \quad \eta(2 i)=\frac{1}{2^{\frac{3}{8}}} \cdot \Omega_{-4}^{\frac{1}{2}}, \quad \eta(4 i)=\frac{(\sqrt{2}-1)^{\frac{1}{4}}}{2^{\frac{13}{16}}} \cdot \Omega_{-4}^{\frac{1}{2}} .
$$

We shall make use of these formulas to prove Corollary 1.2 .

3.2. Modular Forms on $\Gamma_{0}(4)$. Here we recall standard facts about modular forms on $\Gamma_{0}(4)$. Recall that the theta function given by

$$
\theta(\tau):=\sum_{n=-\infty}^{\infty} q^{n^{2}}
$$

is a weight $\frac{1}{2}$ modular form on $\Gamma_{0}(4)$, and that the weight 2 Eisenstein series

$$
F(\tau):=\sum_{n=0}^{\infty} \sigma_{1}(2 n+1) q^{2 n+1}
$$

is a modular form on $\Gamma_{0}(4)$ as well, where $\sigma_{1}(n)$ denotes the sum of the positive divisors of $n$. It is known that every modular form on $\mathrm{SL}_{2}(\mathbb{Z})$ and $\Gamma_{0}(4)$ can be expressed as a rational function in $\eta(\tau), \eta(2 \tau)$, and $\eta(4 \tau)$ (see [8, Theorem 1.67] for $\mathrm{SL}_{2}(\mathbb{Z})$ and [7] for $\Gamma_{0}(4)$ ). In the case of $\Gamma_{0}(4)$, this fact relies on the observation that $F(\tau)$ and $\theta(\tau)$ are given in terms of Dedekind eta-quotients in the following way:

$$
\theta(\tau)=\frac{\eta(2 \tau)^{5}}{\eta(\tau)^{2} \eta(4 \tau)^{2}}, \quad F(\tau)=\frac{\eta(4 \tau)^{8}}{\eta(2 \tau)^{4}} .
$$

It is also very well known that the two Eisenstein series $E_{4}(\tau)$ and $E_{6}(\tau)$ generate the algebra of all modular forms on $\mathrm{SL}_{2}(\mathbb{Z})$ (for example, see [8, Theorem 1.23]). The analogous statement for modular forms on $\Gamma_{0}(4)$ involves the forms $F(\tau)$ and $\theta(\tau)$. Namely, the following complete description of the spaces $M_{k}\left(\Gamma_{0}(4), \psi_{k}\right)$ for $k \in \frac{1}{2} \mathbb{N}$ and

$$
\psi_{k}:= \begin{cases}\chi_{0}, & \text { if } k \in 2 \mathbb{Z} \text { or } k \in \frac{1}{2}+\mathbb{Z}, \\ \left(\frac{-4}{\bullet}\right), & \text { if } k \in 1+2 \mathbb{Z}\end{cases}
$$

where $\chi_{0}$ is the trivial character, is proved in [4,6]. As a graded algebra, we have that

$$
\bigoplus_{k \in \frac{1}{2} \mathbb{Z}} M_{k}\left(\Gamma_{0}(4), \psi_{k}\right) \cong \mathbb{C}[F, \theta] .
$$


Moreover, we have the following proposition (see [7, Corollary 3.3]) describing canonical representations of modular forms on $\Gamma_{0}(4)$ in terms of $F(\tau)$ and $\theta(\tau)$.

Proposition 3.2. If $k \in \frac{1}{2} \mathbb{N}$, then each $f(\tau) \in M_{k}\left(\Gamma_{0}(4), \psi_{k}\right)$ has a unique expansion in terms of $F(\tau)$ and $\theta(\tau)$ of the form

$$
f(\tau)=\sum_{j=0}^{[k / 2]} \alpha_{k}(j) F(\tau)^{j} \theta(\tau)^{2 k-4 j} .
$$

Moreover, $f(\tau)$ is a cusp form if and only if the coefficients $\alpha_{k}(j)$ satisfy:

(i) $\alpha_{k}(0)=0$,

(ii) $\alpha_{k}(k / 2)=0$ when $k \in 2 \mathbb{Z}$, and

(iii) $\sum_{j=0}^{[k / 2]} \alpha_{k}(j)\left(\frac{1}{16}\right)^{j}=0$.

Combining the above decomposition in terms of $F(\tau)$ and $\theta(\tau)$ for the cusp form $T_{2 k}(\tau)$ with the eta-quotients in (3.2), we obtain new expressions for the series $\mathcal{G}_{2 k}(q)$ in Section 4 .

\section{Proof of Theorem 1.1 and Corollary 1.2}

We may write the product on the right hand side of Theorem 2.1 in terms of eta-quotients as follows:

$$
\mathcal{Z}(2 k) \cdot q^{k} \psi\left(q^{2}\right)^{4 k}=\mathcal{Z}(2 k) \cdot q^{k} \prod_{n=1}^{\infty} \frac{\left(1-q^{4 n}\right)^{4 k}}{\left(1-q^{4 n-2}\right)^{4 k}}=\mathcal{Z}(2 k) \cdot \frac{\eta(4 \tau)^{8 k}}{\eta(2 \tau)^{4 k}} .
$$

Now, by $(3.3)^{2}$ we can express the cusp form $T_{2 k}(\tau)$ as

$$
T_{2 k}(\tau)=\sum_{j=0}^{k} \alpha_{2 k}(j) F(\tau)^{j} \theta(\tau)^{4 k-4 j}
$$

and by (3.2) we can write (4.2) in terms of eta-quotients in the following way:

$$
T_{2 k}(\tau)=\frac{\eta(2 \tau)^{20 k}}{\eta(\tau)^{8 k} \eta(4 \tau)^{8 k}} \sum_{j=0}^{k} \alpha_{2 k}(j) \cdot \frac{\eta(4 \tau)^{16 j} \eta(\tau)^{8 j}}{\eta(2 \tau)^{24 j}} .
$$

By Proposition 3.2 (i) and (ii), we may simplify this expression to

$$
T_{2 k}(\tau)=\frac{\eta(2 \tau)^{20 k}}{\eta(\tau)^{8 k} \eta(4 \tau)^{8 k}} \sum_{j=1}^{k-1} \alpha_{2 k}(j) \cdot \frac{\eta(4 \tau)^{16 j} \eta(\tau)^{8 j}}{\eta(2 \tau)^{24 j}} .
$$

Now, combining (4.1) with (4.3), we see that the series $\mathcal{G}_{2 k}(q)$ can be expressed as

$$
\mathcal{G}_{2 k}(q)=\mathcal{Z}(2 k) \cdot \frac{\eta(4 \tau)^{8 k}}{\eta(2 \tau)^{4 k}}+\frac{\eta(2 \tau)^{20 k}}{\eta(\tau)^{8 k} \eta(4 \tau)^{8 k}} \sum_{j=1}^{k-1} \alpha_{2 k}(j) \cdot \frac{\eta(4 \tau)^{16 j} \eta(\tau)^{8 j}}{\eta(2 \tau)^{24 j}}
$$

\footnotetext{
${ }^{2}$ The weights in Theorem 1.1 are $2 k$ as opposed to $k$ in the section above.
} 
From the above expression for $\mathcal{G}_{2 k}(q)$, it is clear that if $k \geq 1$, then the $\alpha_{2 k}(j)$ are the unique rational numbers such that

$$
\sum_{j=1}^{k-1} \alpha_{2 k}(j) \cdot \frac{\eta(4 \tau)^{16 j} \eta(\tau)^{8 j}}{\eta(2 \tau)^{24 j}}=\left(\mathcal{G}_{2 k}(q)-\mathcal{Z}(2 k) \cdot \frac{\eta(4 \tau)^{8 k}}{\eta(2 \tau)^{4 k}}\right) \cdot \frac{\eta(\tau)^{8 k} \eta(4 \tau)^{8 k}}{\eta(2 \tau)^{20 k}}
$$

This implies the definition for the $\alpha_{2 k}(j)$ in (11.6) .

Theorem 3.1 along with (4.4) immediately imply that evaluations of the Goswami-Sun series at $\mathrm{CM}$ points $\tau \in \mathbb{H} \cap \mathbb{Q}(\sqrt{D})$ give values in $\overline{\mathbb{Q}} \cdot \Omega_{D}^{2 k}$, which proves Theorem 1.1 because

$$
\omega_{D}^{2 k}=2^{k}|D|^{k} \cdot \Omega_{D}^{2 k}
$$

Proof of Corollary 1.2. If $D=-4$, then $\mathbb{Q}(\sqrt{D})=\mathbb{Q}(i)$ and by (1.4) we have

$$
\omega_{-4}=\frac{1}{\sqrt{\pi}} \cdot \frac{\Gamma\left(\frac{1}{4}\right)}{\Gamma\left(\frac{3}{4}\right)}
$$

We apply the functional equation of the $\Gamma$-function to rewrite $\omega_{-4}$ as

$$
\omega_{-4}=\frac{\Gamma\left(\frac{1}{4}\right)^{2}}{\sqrt{2} \pi^{3 / 2}} .
$$

In particular, applying the values of $\eta(\tau)$ in (3.1) to all of the eta-functions in (4.4), we evaluate $\mathcal{G}_{2 k}\left(e^{2 \pi i \tau}\right)$ at $\tau=\frac{i}{2}$ and $\tau=i$ to obtain the values in Corollary 1.2.

\section{ExAmples}

Example 5.1. If $k=3$, then Theorem 1.1 becomes

$$
\mathcal{G}_{6}(q)=\mathcal{Z}(6) \cdot \frac{\eta(4 \tau)^{24}}{\eta(2 \tau)^{12}}+\frac{\eta(2 \tau)^{60}}{\eta(\tau)^{24} \eta(4 \tau)^{24}}\left(\alpha_{6}(1) \cdot \frac{\eta(4 \tau)^{16} \eta(\tau)^{8}}{\eta(2 \tau)^{24}}+\alpha_{6}(2) \cdot \frac{\eta(4 \tau)^{32} \eta(\tau)^{16}}{\eta(2 \tau)^{48}}\right)
$$

and we calculate that $\alpha_{6}(1)=1$ and $\alpha_{6}(2)=-16$. Then we have that

$$
\mathcal{G}_{6}(q)=\mathcal{Z}(6) \cdot \frac{\eta(4 \tau)^{24}}{\eta(2 \tau)^{12}}+\frac{\eta(2 \tau)^{60}}{\eta(\tau)^{24} \eta(4 \tau)^{24}}\left(\frac{\eta(4 \tau)^{16} \eta(\tau)^{8}}{\eta(2 \tau)^{24}}-16 \cdot \frac{\eta(4 \tau)^{32} \eta(\tau)^{16}}{\eta(2 \tau)^{48}}\right) .
$$

Corollary 1.2 in this case gives

$$
\mathcal{G}_{6}\left(e^{-\pi}\right)=\left(\frac{\mathcal{Z}(6)}{2^{21}}+\frac{1}{2^{12}}\right) \cdot\left(\frac{\Gamma\left(\frac{1}{4}\right)^{4}}{\pi^{3}}\right)^{3}=0.0633804556 \ldots
$$

and

$$
\mathcal{G}_{6}\left(e^{-2 \pi}\right)=\left(\frac{\mathcal{Z}(6)(\sqrt{2}-1)^{6}}{2^{27}}+\frac{1-(\sqrt{2}-1)^{4}}{2^{19}(\sqrt{2}-1)^{2}}\right) \cdot\left(\frac{\Gamma\left(\frac{1}{4}\right)^{4}}{\pi^{3}}\right)^{3}=0.0018690318 \ldots
$$

Example 5.2. If $k=4$, then Theorem 1.1 becomes

$$
\begin{aligned}
\mathcal{G}_{8}(q) & =\mathcal{Z}(8) \cdot \frac{\eta(4 \tau)^{32}}{\eta(2 \tau)^{16}} \\
+ & \frac{\eta(2 \tau)^{80}}{\eta(\tau)^{32} \eta(4 \tau)^{32}}\left(\alpha_{8}(1) \cdot \frac{\eta(4 \tau)^{16} \eta(\tau)^{8}}{\eta(2 \tau)^{24}}+\alpha_{8}(2) \cdot \frac{\eta(4 \tau)^{32} \eta(\tau)^{16}}{\eta(2 \tau)^{48}}+\alpha_{8}(3) \cdot \frac{\eta(4 \tau)^{48} \eta(\tau)^{24}}{\eta(2 \tau)^{72}}\right)
\end{aligned}
$$


and we calculate that $\alpha_{8}(1)=0, \alpha_{8}(2)=128$, and $\alpha_{8}(3)=-2048$. Then we have that

$$
\mathcal{G}_{8}(q)=\mathcal{Z}(8) \cdot \frac{\eta(4 \tau)^{32}}{\eta(2 \tau)^{16}}+\frac{\eta(2 \tau)^{80}}{\eta(\tau)^{32} \eta(4 \tau)^{32}}\left(128 \cdot \frac{\eta(4 \tau)^{32} \eta(\tau)^{16}}{\eta(2 \tau)^{48}}-2048 \cdot \frac{\eta(4 \tau)^{48} \eta(\tau)^{24}}{\eta(2 \tau)^{72}}\right)
$$

Corollary 1.2 in this case gives

$$
\mathcal{G}_{8}\left(e^{-\pi}\right)=\left(\frac{\mathcal{Z}(8)}{2^{28}}+\frac{1}{2^{12}}\right) \cdot\left(\frac{\Gamma\left(\frac{1}{4}\right)^{4}}{\pi^{3}}\right)^{4}=0.2980189122 \ldots
$$

and

$$
\mathcal{G}_{8}\left(e^{-2 \pi}\right)=\left(\frac{\mathcal{Z}(8)(\sqrt{2}-1)^{8}}{2^{36}}+\frac{1-(\sqrt{2}-1)^{4}}{2^{21}}\right) \cdot\left(\frac{\Gamma\left(\frac{1}{4}\right)^{4}}{\pi^{3}}\right)^{4}=0.0004465790 \ldots
$$

\section{REFERENCES}

[1] B. C. Berndt. Ramanujan's Notebooks, Part V. Springer-Verlag, New York (1998).

[2] J. H. Bruinier, G. van der Geer, G. Harder, and D. Zagier. The 1-2-3 of Modular Forms. Springer-Verlag, Berlin (2008).

[3] S. Chowla and A. Selberg. On Epstein's zeta-function. J. Reine Angew. Math. 227 (1967), pp. 86-110.

[4] H. Cohen. Sums involving the values at negative integers of L-functions of quadratic characters. Math. Ann. 217 (1975), pp. 271-285.

[5] A. Goswami. A $q$-analogue for Euler's $\zeta(2 k)=\frac{(-1)^{k+1} 2^{2 k} B_{2 k} \pi^{2 k}}{2(2 k) !}$. Preprint, arXiv:1803.02467.

[6] N. Koblitz. Introduction to elliptic curves and modular forms. Springer-Verlag, New York (1984).

[7] K. Ono. Differential endomorphisms for modular forms on $\Gamma_{0}(4)$. Symbolic Computation, Number Theory, Special Functions, Physics, and Combinatorics, Kluwer Academic Publishers (1999) pp. 223-230.

[8] K. Ono. The Web of Modularity: Arithmetic of the Coefficients of Modular Forms and q-series. AMS and CBMS, Providence, RI (2004).

[9] A. van der Poorten and K. Williams. Values of the Dedekind eta function at quadratic irrationalities. Canad. J. Math. Vol. 51 (1), 1999, pp. 176-224.

[10] Z.-W. Sun. Two $q$-analogues of Euler's formula $\zeta(2)=\pi^{2} / 6$. Preprint, arXiv:1802.01473

Department of Mathematics and Computer Science, Emory University, Atlanta, Ga 30322

E-mail address: madeline.locus@emory.edu

Department of Mathematics and Computer Science, Emory University, Atlanta, Ga 30322

E-mail address: ono@mathcs.emory.edu 